\title{
Mindfulness-based interventions for mental well- being among people with multiple sclerosis: a systematic review and meta-analysis of randomised controlled trials
}

\author{
Robert Simpson, ${ }^{1}$ Sharon Simpson, ${ }^{1}$ Nitish Ramparsad, ${ }^{2}$ Margaret Lawrence, ${ }^{3}$ \\ Jo Booth, ${ }^{3}$ Stewart W Mercer ${ }^{4}$
}

- Additional material is published online only. To view please visit the journal online (http://dx.doi.org/10.1136/ jnnp-2018-320165)

${ }^{1}$ Institute of Health and Wellbeing, University of Glasgow, Glasgow, UK ${ }^{2}$ Robertson Centre for Biostatistics, University of Glasgow, Glasgow, UK ${ }^{3} S$ chool of Health and Life Sciences, Glasgow Caledonian University, Glasgow, UK ${ }^{4}$ General Practice and Primary Care, University of Glasgow, Glasgow, UK

\section{Correspondence to} Dr Robert Simpson, Institute of Health and Wellbeing, University of Glasgow, Glasgow G12 8QQ, UK; Robert.Simpson@glasgow. ac.uk

Received 14 December 2018 Revised 17 May 2019 Accepted 22 May 2019 Published Online First 13 June 2019

Check for updates

(C) Author(s) (or their employer(s)) 2019. No commercial re-use. See rights and permissions. Published by BMJ.

To cite: Simpson R, Simpson S, Ramparsad N, et al. J Neurol Neurosurg Psychiatry 2019:90:1051-1058.

\begin{abstract}
Objective Impairment of mental well-being (anxiety, depression, stress) is common among people with multiple sclerosis (PwMS). Treatment options are limited, particularly for anxiety. The aim of this study was to update our previous systematic review (2014) and evaluate via meta-analysis the efficacy of mindfulnessbased interventions (MBIs) for improving mental wellbeing in PwMS.
\end{abstract}

Methods Systematic searches for eligible randomised controlled trials (RCTs) were carried out in seven major databases (November 2017, July 2018), using medical subject headings and key words. Studies were screened, data extracted, quality appraised and analysed by two independent reviewers, using predefined criteria. Study quality was assessed using the Cochrane Collaboration risk of bias tool. Mental well-being was the primary outcome. Random effects model meta-analysis was performed, with effect size reported as standardised mean difference (SMD).

Results Twelve RCTs including 744 PwMS were eligible for inclusion in the systematic review, eight had data extractable for meta-analysis; $n=635$. Ethnicity, socioeconomic status, comorbidity and disability were inconsistently reported. MBIs varied from manualised to tailored versions, lasting 6-9 weeks, delivered individually and via groups, both in person and online. Overall SMD for mental well-being (eight studies) was $0.40(0.28-0.53), p<0.01, l^{2}=28 \%$; against active comparators only (three studies) SMD was 0.17 $(0.01-0.32), p<0.05, I^{2}=0 \%$. Only three adverse events were reported.

Conclusions MBls are effective at improving mental well-being in PwMS. More research is needed regarding optimal delivery method, cost-effectiveness and comparative-effectiveness.

PROSPERO registration number CRD42018093171.

\section{BACKGROUND}

People with multiple sclerosis (PwMS) often describe the condition as stressful. ${ }^{1}$ Mental health comorbidity is common; ${ }^{2}$ anxiety and depression three times as frequent compared with population norms ${ }^{2}$ and are associated with higher levels of somatic symptoms, increased suicidality, lower quality of life and greater social problems. ${ }^{3}$
Very little evidence exists on the optimal treatment for impaired mental well-being in PwMS. Systematic review and meta-analytic evidence supports cognitive behavioural therapy (CBT) for both stress and depression in PwMS, but effective treatments for anxiety are lacking. ${ }^{45}$

Mindfulness-based interventions (MBIs) are complex interventions ${ }^{6}$ increasingly used in healthcare. MBIs have high quality evidence for treating stress, anxiety and recurrent depression in the general population ${ }^{7}$ and thus might also help PwMS with impaired mental well-being. In 2014, our previous systematic review found preliminary evidence from two randomised controlled trials (RCTs) and a controlled trial to support MBIs as a potential treatment for anxiety and depression in PwMS. ${ }^{8}$ Due to heterogeneity between study type, populations, interventions and outcomes, meta-analysis was not possible, nor was the optimal MBI for PwMS clear. Since 2014 several more RCTs have been published.

The aim of this review is to undertake a meta-analysis of RCT evidence for MBIs in improving mental well-being in people with MS.

\section{METHODS}

Eligibility for inclusion

Based on the Study design, Participants, Interventions, Outcomes (SPIO) model (a derivative of Population, Intervention, Comparator, Outcome, Study [PICOS] $){ }^{9}$ eligibility included: RCTs of patients with any diagnosis of MS, aged $=/>18$, any type of MBI (including core components of mindful breath awareness, body awareness and mindful movement), with outcomes focused primarily on impact on mental well-being.

\section{Search strategy}

We used our previous systematic review search strategy in: MEDLINE, EMBASE, CINAHL, Cochrane Central Register of Controlled Trials, AMED and PsycInfo. The 'years' delimiter was 2000-2018; our previous systematic review found the first study in this area was in 2000. We also searched ProQuest Dissertations \& Theses Database, reference lists from key papers, contacted relevant experts and searched the grey literature. The initial search was in November 2017, updated 
in July 2018. Online supplementary file 1 contains the search strategy formatted for MEDLINE.

\section{Study selection, storage and screening}

Search results were first imported into COVIDENCE, a systematic review data storage software package. Two independent reviewers (RS, SS) screened study title/abstracts for potential eligibility using keywords like 'mindfulness' and 'multiple sclerosis' (MS). Selected studies were then assessed further by two independent reviewers (RS, JB) against SPIO criteria to determine definitively eligibility. A third-party senior reviewer adjudicated any disagreements (SM).

\section{Data collection/data items}

Once the final list of studies for inclusion was agreed, data was extracted and expanded to ensure all Consolidated Standards of Reporting Trials (CONSORT) ${ }^{10}$ and Template for Intervention Description and Replication (TIDieR) ${ }^{11}$ checklist items were included (online supplementary file 2).

\section{Quality appraisal}

The Cochrane Collaboration's assessment tool ${ }^{12}$ was used to summarise the risk of bias for major outcomes in selected studies for individual outcomes, graded as high, unclear or low risk, assessing sequence generation, allocation concealment, participant blinding, personnel and outcome assessor blinding, completeness of outcome data, selective outcome reporting and any other sources of bias. Overall risk of bias for each study was also graded as:

Low $=$ low risk of bias for all key domains

Unclear $=$ low or unclear risk of bias for all key domains

High $=$ high risk of bias for one or more key domains.

\section{Principal summary measures}

The 'Primary outcome' was identified as mental well-being (anxiety \pm depression \pm stress). The main outcome measure was taken as the last follow-up at which data were reported for that outcome. All main outcome measures were reported as continuous measures and their mean, SD and number of subjects for each treatment group were extracted. The unbiased standardised mean difference (SMD) was calculated, whereby a positive SMD reflects a difference in favour of MBI. Where papers reported effect estimates from adjusted regression models, these were extracted as the SMD.

\section{Synthesis of results}

We followed the Preferred Reporting Items for Systematic Reviews and Meta-Analyses (PRISMA) ${ }^{13}$ guidance. Due to the wide variety of outcome measures identified and known heterogeneity, random-effects meta-analysis regression model ${ }^{14}$ was used to derive SMD. Estimates are reported along with their corresponding 95\% CI and p values. The $\mathrm{I}^{2}$ statistic was used to assess the variability between studies. ${ }^{15} \mathrm{I}^{2}$ describes the percentage of total variability in the estimates effect size that is attributable to heterogeneity. $\mathrm{I}^{2}$ varies from $0 \%$ (all heterogeneity is due to sampling error) to $100 \%$ (all variability due to true heterogeneity between studies)

Funnel plots and Egger's Test for asymmetry were undertaken to test for publication bias and 'trim and fill' method was undertaken to assess the impact of the bias. ${ }^{16-19}$

All statistical analyses were carried out in R V.3.4.0 and using the meta package. ${ }^{20}$

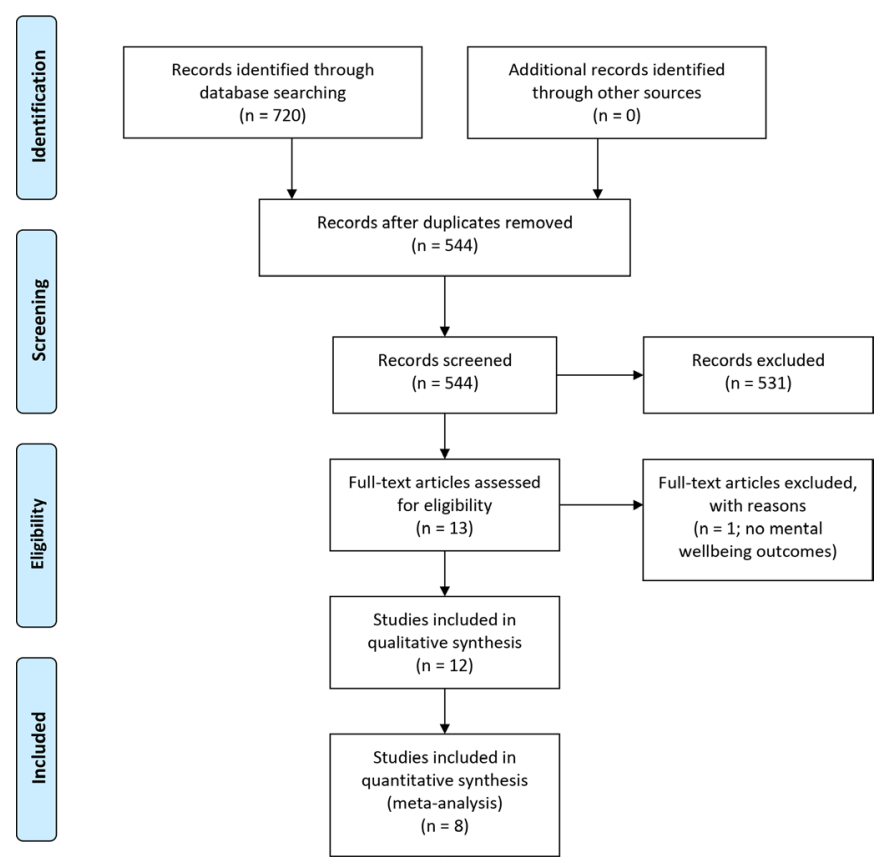

Figure 1 Preferred Reporting Items for Systematic Reviews and MetaAnalyses flow diagram.

\section{RESULTS}

Twelve RCTs were identified as eligible for inclusion in the systematic review. ${ }^{21-32}$ However, only eight studies reported endpoint data that could be included in the meta-analysis. ${ }^{21-24} 28-3032$ (figure 1). Further details were sought from study authors, ${ }^{29} 3132$ but only one ${ }^{32}$ responded.

\section{Study characteristics}

Five studies took place in Iran, ${ }^{23} 25-2729$ three in the UK, ${ }^{22} 2831$ two in Italy, ${ }^{24} 32$ one each in Switzerland ${ }^{30}$ and the USA. ${ }^{21}$ Six studies reported assessing a MBI against usual care, ${ }^{22} 23$ 28-31 in three this was not specified, ${ }^{22-24}$ while three used an active comparator (psychoeducation control). ${ }^{21} 2432$ Six studies were powered. $^{21} 2324293032$ Sample sizes ranged from 24 to 150 (median 49). Eight studies measured outcomes at three time points (baseline, post MBI and at follow-up; range 1 month-1 year), ${ }^{21} 22 \quad 24 \quad 28-32$ while four were pre-post measurements only. $^{2325-27}$ (table 1).

\section{Participant characteristics}

Across the 12 RCTs, the total number of participants was 744 , those in the meta-analysis 635 . Three studies reported ethnicity, $^{21} 2228$ mostly Caucasian. Overall, 76\% $(n=565)$ of study participants were female. Where reported, overall mean age of participants was 41.4 years (age not reported in one study $\left.{ }^{26}\right)$. Only two studies included details on socioeconomic status (SES), ${ }^{22} 25$ three minimal details on employment status. ${ }^{22} 2431$ Ten studies included details on education status, ${ }^{21-23} 25-3032$ with the majority in nine studies ${ }^{21-23} 25-3032$ having completed at least school level education. Most (at least 447 or 60\%) had relapsing-remitting MS, at least $112(15 \%)$ had secondary progressive MS and at least 30 (4\%) had primary progressive MS. Mean Expanded Disability Status Scale (EDSS) was reported in five studies, ${ }^{2122} 242830$ ranging from 2.3 to 6.5. Only one study reported on number of comorbid conditions (mean 2.3, SD 1.7), ${ }^{22}$ with four studies reporting on the active 
Table 1 Study characteristics

\begin{tabular}{|c|c|c|c|c|c|c|}
\hline Study & Country & $\begin{array}{l}\text { Study } \\
\text { design }\end{array}$ & Powered & Comparator & $\begin{array}{l}\text { Sample } \\
\text { size }(n)\end{array}$ & Study attrition (\%) \\
\hline $\begin{array}{l}\text { Mills and\& Allen } \\
\text { et } a l^{31}\end{array}$ & Wales (UK) & $\mathrm{RCT}$ & No & TAU & $n=24$ & $33 \%$ \\
\hline Grossman et $a l^{30}$ & Switzerland & $\mathrm{RCT}$ & Yes & TAU & $n=150$ & $5 \%$ \\
\hline
\end{tabular}

Outcome measures

(others) Data collection

POMS, Standing Baseline, post, 3

balance, Symptom rating months follow-up questionnaire

Grossman et a ${ }^{30}$ Switzerland - RCT $\quad$ Yes $\quad$ TAU $\quad n=150 \quad 5 \%$

CES-D, STAI, MFIS, Baseline, post, 6

HAQUAMS, PQOLC, months follow-up

(Neuropsychology

assessment, goal

attainment)

\begin{tabular}{|c|c|c|c|c|c|c|c|c|}
\hline Bogosian et al ${ }^{28}$ & England (UK) & $\mathrm{RCT}$ & No & TAU & $n=40$ & $5 \%$ & GHQ, HADS, MSIS, FSS & $\begin{array}{l}\text { Baseline, post, } 3 \\
\text { months follow-up }\end{array}$ \\
\hline $\begin{array}{l}\text { Kolahkaj and } \\
\text { Zargar }^{29}\end{array}$ & Iran & $\mathrm{RCT}$ & Yes & TAU & $\mathrm{n}=48$ & $17 \%$ & DASS-21 & $\begin{array}{l}\text { Baseline, post, } 2 \\
\text { months follow-up }\end{array}$ \\
\hline Amiri et $a l^{25}$ & Iran & $\mathrm{RCT}$ & No & Unclear & $\mathrm{n}=40$ & $0 \%$ & STAI, BDI-2, WCST & Baseline, post \\
\hline Mahdavi et al ${ }^{26}$ & Iran & $\mathrm{RCT}$ & No & Unclear & $n=24$ & $0 \%$ & $\begin{array}{l}\text { BAI, BDI-2, FSS, MWQ, } \\
\text { TFI }\end{array}$ & Baseline, post \\
\hline Nejati et $a l^{27}$ & Iran & $\mathrm{RCT}$ & Unclear & Unclear & $n=24$ & $0 \%$ & MSQOL-54, FSS & Baseline, post \\
\hline Bahrani et $a l^{23}$ & Iran & $\mathrm{RCT}$ & Yes & TAU & $n=56$ & $16 \%$ & DASS-21 & Baseline, post \\
\hline Simpson et al ${ }^{22}$ & Scotland (UK) & $\mathrm{RCT}$ & No & TAU & $n=50$ & $12 \%$ & $\begin{array}{l}\text { PSS, EQ5D5L, MSQLI, } \\
\text { MAAS, SCS-sf, ELQ }\end{array}$ & $\begin{array}{l}\text { Baseline, post, } 3 \\
\text { months follow-up }\end{array}$ \\
\hline Carletto et $a{ }^{24}$ & Italy & $\mathrm{RCT}$ & Yes & $\begin{array}{l}\text { Psycho-education } \\
\text { intervention }\end{array}$ & $\mathrm{n}=90$ & $21 \%$ & $\begin{array}{l}\text { BDI-2, BAI, PSS, BIPQ, } \\
\text { FAMS }\end{array}$ & $\begin{array}{l}\text { Baseline, post-BAM, } 6 \\
\text { months post-BAM }\end{array}$ \\
\hline Cavalera et a/ ${ }^{32}$ & Italy & $\mathrm{RCT}$ & Yes & $\begin{array}{l}\text { Psycho-education } \\
\text { intervention }\end{array}$ & $\mathrm{n}=139$ & $39 \%$ & $\begin{array}{l}\text { MSQOL-54, HADS, MOSS, } \\
\text { MFIS, }\end{array}$ & $\begin{array}{l}\text { Baseline, post-, } 6 \\
\text { months post MBI }\end{array}$ \\
\hline Senders et $a l^{21}$ & USA & $\mathrm{RCT}$ & Yes & $\begin{array}{l}\text { Educational control, } \\
\text { matched for time and } \\
\text { attention }\end{array}$ & $n=62$ & $16 \%$ & $\begin{array}{l}\text { PSS, PROMIS, CD-RISC, } \\
\text { PASAT }\end{array}$ & $\begin{array}{l}\text { Baseline, mid- } \\
\text { intervention, } \\
\text { immediately post-, } \\
4,8 \text { and } 12 \text { months } \\
\text { post-MBI }\end{array}$ \\
\hline
\end{tabular}

BAI, Beck anxiety inventory; BAM, body-affective mindfulness; BDI-2, Beck depression inventory-2; BIPQ, Brief illness perception questionnaire; CD-RISC, Connor-Davidson Resilience Scale; CES-D, Center for epidemiological studies depression scale; DASS-21, Depression, Anxiety, and Stress Scale-21; ELQ, Emotional lability questionnaire; EQ-5D5L, EuroQol; FAMS, Functional Assessment of Multiple Sclerosis; FSS, Fatigue severity scale; GHQ, General health questionnaire; HADS, Hospital anxiety and depression scale; HAQUAMS, Hamburg quality of life questionnaire in multiple sclerosis (German); MAAS, Mindful attention awareness scale; MFIS, Modified fatigue impact scale; MOSS, Medical Outcomes Sleep Scale; MSIS, Multiple sclerosis impact scale; MSQLI, Multiple sclerosis quality of life inventory; MSQOL-54, Multiple sclerosis quality of life - 54; MWQ, Meta worry questionnaire; PASAT, Paced Auditory Serial Attention Task; POMS, Profile of mood states; PQOLC, Profile of health related quality of life in chronic disorders (German); PROMIS, Patient-Reported Outcomes Information System; PSS, Perceived stress scale; RCT, Randomised controlled trial; SCS-sf, Self-compassion scale-short form; STAI, Spielberger trait anxiety inventory; TAU, Treatment as usual; TFI, Thought fusion inventory; WCST, Wisconsin card sorting test.

use of disease modifying drugs and/or psychotropic medications $^{21223032}$ (table 2).

\section{Intervention characteristics}

Five studies used mindfulness-based stress reduction (MBSR), 2122293032 two based on MBSR, ${ }^{24} 27$ three mindfulness-based cognitive therapy (MBCT), ${ }^{252628}$ one mindfulness-integrated CBT $(\mathrm{MiCBT})^{23}$ and one mindfulness of movement. ${ }^{31}$ Six studies reported on participant materials. ${ }^{22} 2327283132$ Three studies required a personal intake interview to take part, ${ }^{262730}$ two sought baseline evidence of impaired mental well-being. ${ }^{21}{ }^{28}$ Eight studies reported session content, ${ }^{21-23}$ 25-29 three gave minimal description, ${ }^{30-32}$ one referred to the study protocol. ${ }^{24}$ Seven studies described home practices. ${ }^{21-24} 283031$ Seven studies reported teacher characteristics, ${ }^{21-24} 28-30$ but in two detail was minimal. $^{2329}$ Eleven studies delivered group MBIs, ${ }^{21-3032}$ one was individual. ${ }^{31}$ Two studies used online MBIs. ${ }^{28} 32$ Four studies reported intervention delivery location. ${ }^{22} 282932$ Three studies had nine MBI sessions, ${ }^{21} 240$ eight had eight, ${ }^{22} 2325-2932$ one had six. ${ }^{31}$ Session length ranged from 1 to 3 hours. Class sizes ranged from five to 25 , either one or two instructors present. Six studies modified the MBI for PwMS, 2228 30-32 one study during the course $^{22}$ simplifying mindful movement. Seven studies monitored treatment adherence (session attendance \pm home practice), 21222528 30-32 four considered fidelity assessment, ${ }^{22} 252832$ two recording/checking sessions. ${ }^{25} 28$ Ten studies delivered core MBI components. ${ }^{21-25}$ 27-31 One study removed mindful movement. ${ }^{28}$ Three included an MBSR day retreat at week six. ${ }^{21} 2430$ (For TIDieR checklist items, see online supplementary file 3.)

\section{Outcome characteristics}

Eleven studies measured MBI effect on anxiety, ${ }^{21-26}$ 28-31 eleven on depression, ${ }^{21-2628-31}$ six on stress. ${ }^{21-2428} 29$ One assessed likely cost-effectiveness, finding $87 \%$ probability of savings on service costs and improved outcomes. ${ }^{28}$ Three studies reported mean daily home practice $\left(32,29.2,32.5 \mathrm{~min}^{22} 3031\right)$; another median $\left(38 \mathrm{~min} /\right.$ day; range $\left.14-80^{21}\right)$. Study attrition ranged from $0 \%$ to $39 \%$.

\section{Meta-analysis}

Effect of MBIs on mental well-being measures

Eleven studies investigated MBI effect on mental wellbeing, ${ }^{21-2628-32}$ however only eight ${ }^{21-2428-30} 32$ reported extractable endpoint data. Meta-analysis showed an overall SMD of $0.40 \quad(0.28-0.53 ; \mathrm{p}<0.001), \quad \mathrm{I}^{2}=28 \% \quad$ (low heterogeneity) (figure 2); against active comparators SMD was 0.17 (0.010.32 ), $\mathrm{p}<0.05, \mathrm{I}^{2}=0 \%$ (low heterogeneity) (figure 3 ). Eight studies evaluated MBI effect on anxiety, ${ }^{21-2428-3032}$ where the SMD was $0.35(0.15-0.55), \mathrm{I}^{2}=25 \%$ (low heterogeneity). Eight 


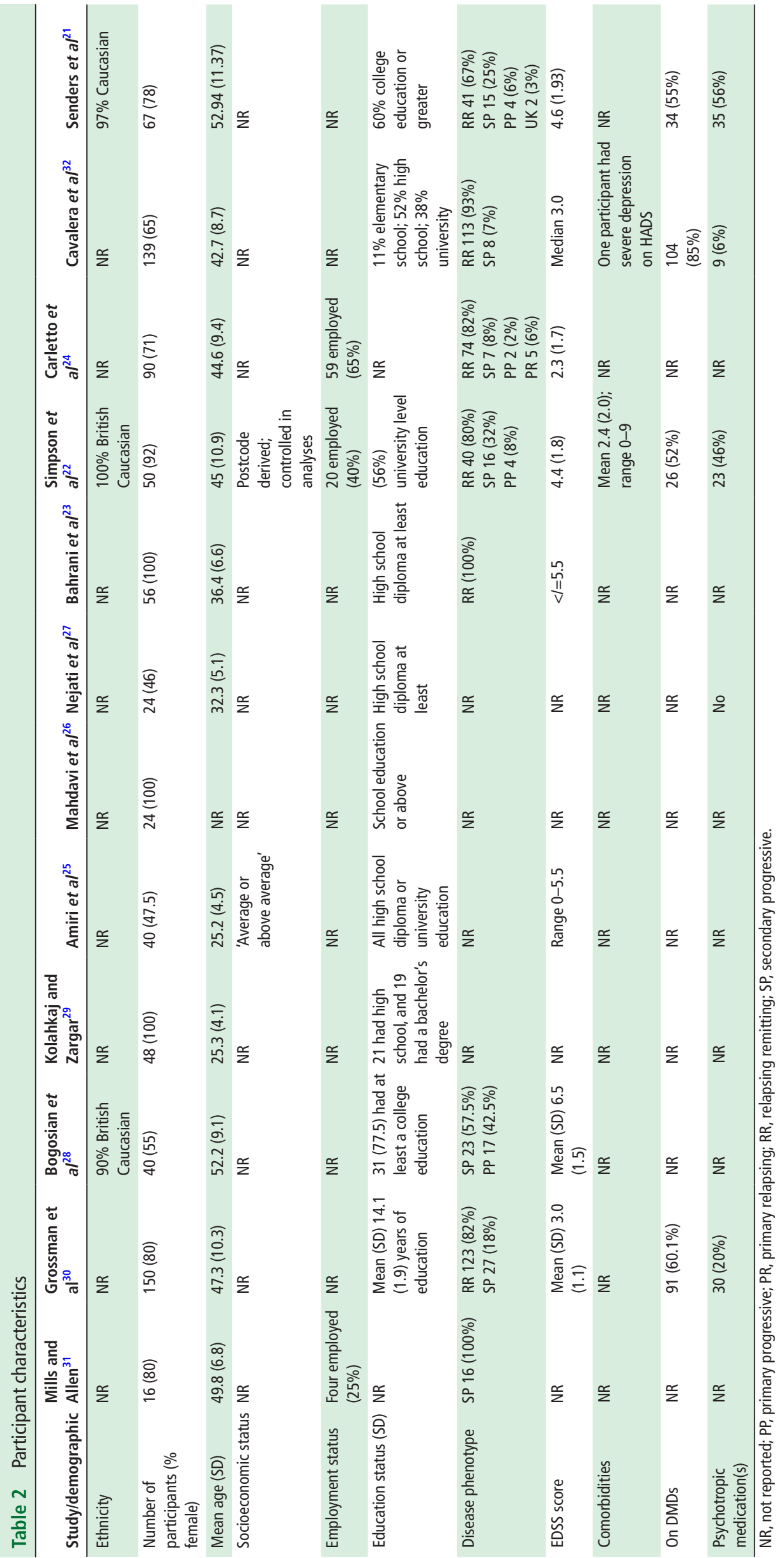




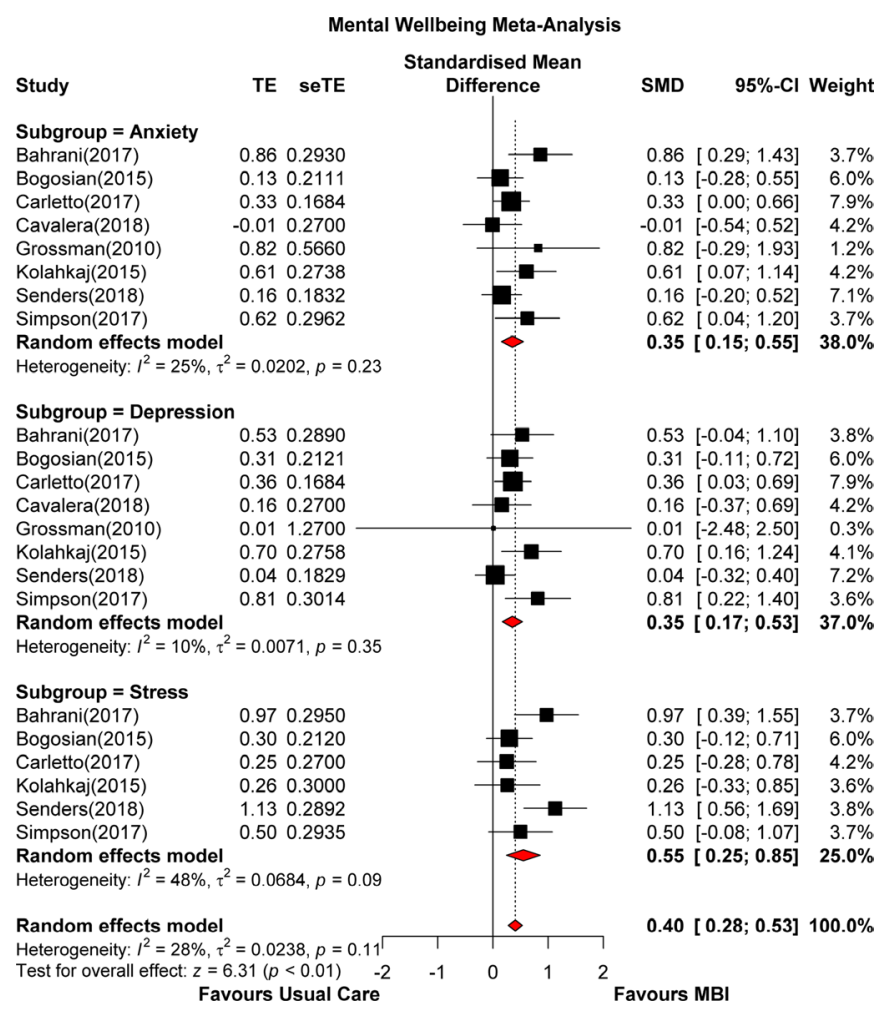

Figure 2 Mental well-being (all comparators) forest plot. MBI, mindfulness-based intervention; SMD, standardised mean difference; seTE, standard error of treatment estimate; TE, estimated treatment effect

studies evaluated MBI effect on depression, ${ }^{21-2428-3032}$ where the SMD was 0.35 (0.17-0.53), $\mathrm{I}^{2}=10 \%$ (low heterogeneity). Six studies evaluated MBI effect on stress, ${ }^{21-2428} 29$ where the SMD was $0.55(0.25-0.85), \mathrm{I}^{2}=48 \%$ (moderate heterogeneity).

Heterogeneity and publication bias

Heterogeneity, $\mathrm{I}^{2}$, among the studies was at $28 \%$ (low heterogeneity).

There was no evidence of publication bias from the funnel plot (figure 4) and Egger's Test of asymmetry confirmed that there was no evidence of asymmetry in the funnel plot. However, this was exactly on the threshold at $p=0.05$. When the trim and

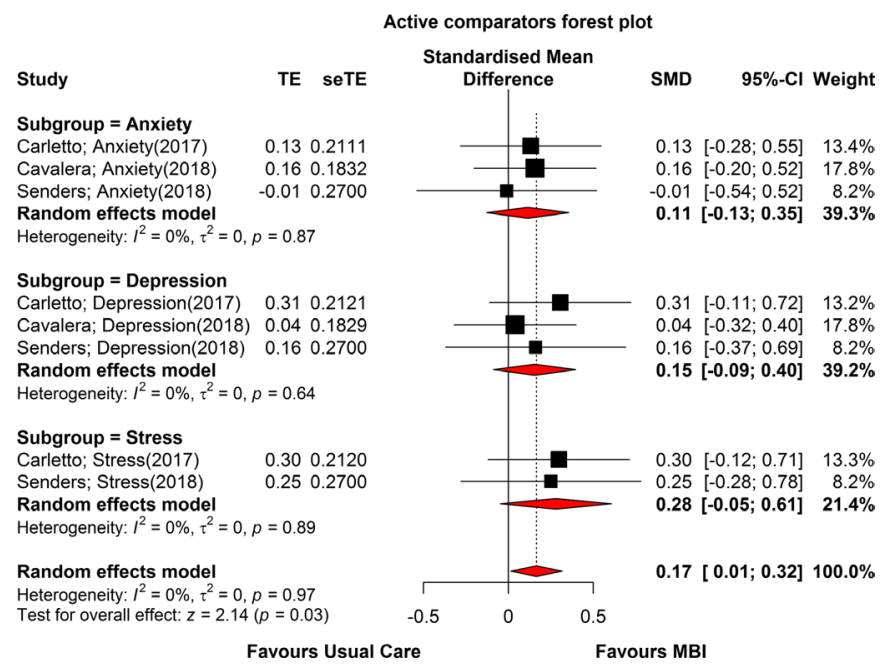

Figure 3 Mental well-being (active comparators only) forest plot. MBI, mindfulness-based intervention; SMD, standardised mean difference.
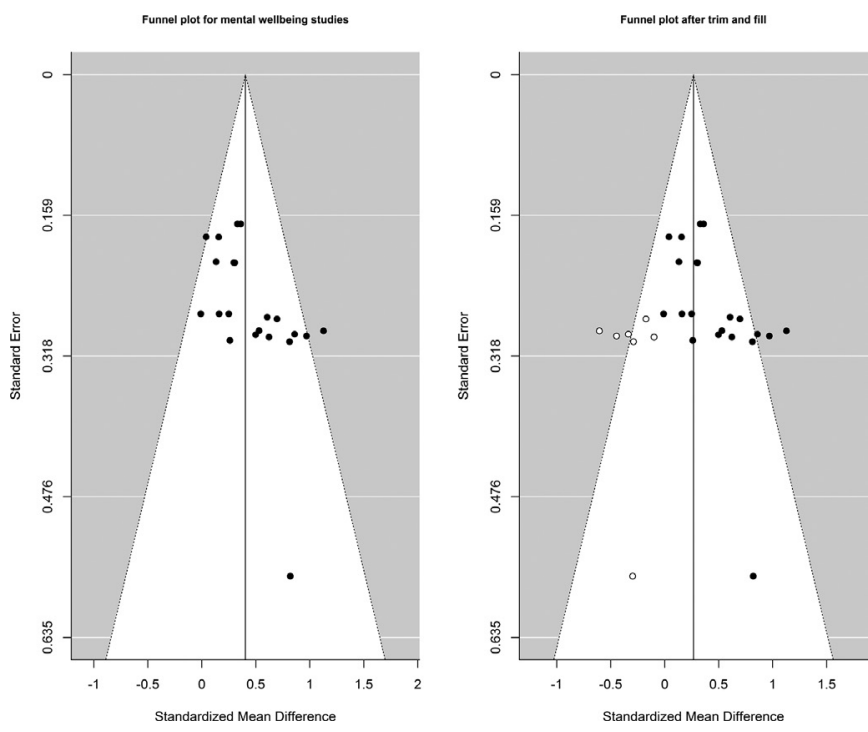

Figure 4 Funnel plot, trim and fill.

fill method was implemented, the estimated number of missing studies was seven. After adjustment for 'missing' studies, the pooled SMD estimate was $0.27(0.12-0.42$; $\mathrm{p}<0.001)$.

Outcomes by intervention type

The largest overall effects were reported for $\mathrm{MiCBT}^{23}$ SMD $0.80(0.48-1.12), I^{2}=0 \%$, but this was a pre- post- RCT $(n=56)$, vs usual care. Overall effects for MBCT vs usual care came from a small study ${ }^{28}(n=40)$, where SMD was $0.78(0.45,1.11)$, $\mathrm{I}^{2}=0 \%$. In another study ${ }^{24}(\mathrm{n}=90)$, compared with a psychoeducation control, body-affective mindfulness had an overall SMD of $0.24(0.00-0.48), \mathrm{I}^{2}=0 \%$. From the five studies 2122293032 with extractable endpoint data that used MBSR (total $n=449$ ), overall SMD was $0.29(0.15-0.42), \mathrm{I}^{2}=0 \%$, three studies 222930 comparing MBSR against usual care, two ${ }^{21} 32$ against psychoeducation controls.

\section{Study quality}

Study quality varied widely. Poor reporting frequently hampered assessment. The highest quality studies derived from Europe and North America. Random sequence generation was well described in nine studies. ${ }^{21-24}$ 27-30 32 Allocation concealment was assessed low risk in six studies, ${ }^{21-24} 2830$ and unclear in the remaining six..$^{25-27} 293132$ Six studies described blinding of assessors, ${ }^{21-24} 2830$ while six reported outcome assessor blinding. ${ }^{21-24} 2830$ Five studies were adjudged low risk of bias for incomplete outcome reporting, ${ }^{21} 22283032$ while selective outcome reporting was adjudged high risk in one. ${ }^{31}$ Overall, five studies were adjudged low risk of bias, ${ }^{2122242830}$ two unclear, ${ }^{23} 32$ five high. $^{252931}$ (table 3). Justifications for risk of bias scores are available in online supplementary file 4 .

When results were pooled, studies adjudged high risk of bias reported the largest overall treatment effects. Figure 5 shows the SMD for all analysable trials grouped by their risk of bias (high, unclear and low) ratings. High risk of bias $(\mathrm{N}=3)$ SMD was 0.64 $(0.31-0.98 ; \mathrm{p}=0.002)$, low risk of bias $(\mathrm{N}=28) \mathrm{SMD}$ was 0.32 $(0.24-0.41 ; \mathrm{p}<0.0001)$ and unclear risk of bias $(\mathrm{N}=7)$ SMD was $0.35(0.08-0.62 ; p=0.01)$. The overall risk of bias analysis showed effect estimates did not significantly differ between risk of bias groups, $\mathrm{p}=0.20$. 
Table 3 Risk of bias

\begin{tabular}{|c|c|c|c|c|c|c|c|c|c|c|c|c|}
\hline Study/risk area & $\begin{array}{l}\text { Mills and } \\
\text { Allen }^{31}\end{array}$ & $\begin{array}{l}\text { Grossmann } \\
\text { et } \mathrm{al}^{30}\end{array}$ & $\begin{array}{l}\text { Bogosian } \\
\text { et } \mathrm{al}^{28}\end{array}$ & $\begin{array}{l}\text { Kolahkaj } \\
\& \\
\text { Zargar }^{29}\end{array}$ & $\begin{array}{l}\text { Amiri et } \\
a l^{25}\end{array}$ & $\begin{array}{l}\text { Mahdavi } \\
\text { et } a l^{26}\end{array}$ & $\begin{array}{l}\text { Nejati et } \\
a^{27}\end{array}$ & $\begin{array}{l}\text { Bahrani } \\
\text { et } a l^{23}\end{array}$ & $\begin{array}{l}\text { Simpson } \\
\text { et } a l^{22}\end{array}$ & $\begin{array}{l}\text { Carletto } \\
\text { et } a l^{24}\end{array}$ & $\begin{array}{l}\text { Cavelera } \\
\text { et } a l^{32}\end{array}$ & $\begin{array}{l}\text { Senders } \\
\text { et } a l^{21}\end{array}$ \\
\hline $\begin{array}{l}\text { Random sequence } \\
\text { generation (selection bias) }\end{array}$ & Unclear & Low & Low & Low & Unclear & Unclear & Low & Low & Low & Low & Low & Low \\
\hline $\begin{array}{l}\text { Allocation concealment } \\
\text { (selection bias) }\end{array}$ & Unclear & Low & Low & Unclear & Unclear & Unclear & Unclear & Low & Low & Low & Unclear & Low \\
\hline $\begin{array}{l}\text { Blinding of assessors } \\
\text { (performance bias) }\end{array}$ & Unclear & Low & Low & Unclear & Unclear & Unclear & Unclear & Low & Low & Low & Unclear & Low \\
\hline $\begin{array}{l}\text { Blinding of outcome } \\
\text { assessment (detection bias) } \\
\text { (patient reported outcomes) }\end{array}$ & High & Low & Low & Unclear & Unclear & Unclear & Unclear & Low & Low & Low & Unclear & Low \\
\hline $\begin{array}{l}\text { Incomplete outcome data } \\
\text { addressed (attrition bias) }\end{array}$ & Unclear & Low & Low & High & High & Unclear & Unclear & Unclear & Low & Low & Unclear & Low \\
\hline $\begin{array}{l}\text { Selective outcome reporting } \\
\text { (reporting bias) }\end{array}$ & High & Low & Low & Low & Low & Low & Low & Low & Low & Low & Low & Low \\
\hline $\begin{array}{l}\text { Other sources of bias (ie, } \\
\text { baseline bias) }\end{array}$ & Unclear & Low & Low & High & Unclear & High & High & Low & Low & Low & Low & Low \\
\hline Overall risk of bias & High & Low & Low & High & High & High & High & Unclear & Low & Low & Unclear & Low \\
\hline
\end{tabular}

\section{Meta-regression}

A meta-regression was fitted to analyse the association between predictors and effect estimate. A backward manual selection process was used with intervention type, risk of bias, mean age, gender and EDSS scores as covariates in the model. Covariates were sequentially excluded based on $\mathrm{p}$ values (significance level at $5 \%$ ) to obtain a final model. MBCT and high risk of bias were found to be significant predictors of the effect estimate (table 4)

\section{Adverse events}

Discreet adverse events were described in two studies; ${ }^{21} 22$ an exacerbation of chronic neuropathic pain during the 'Raisin

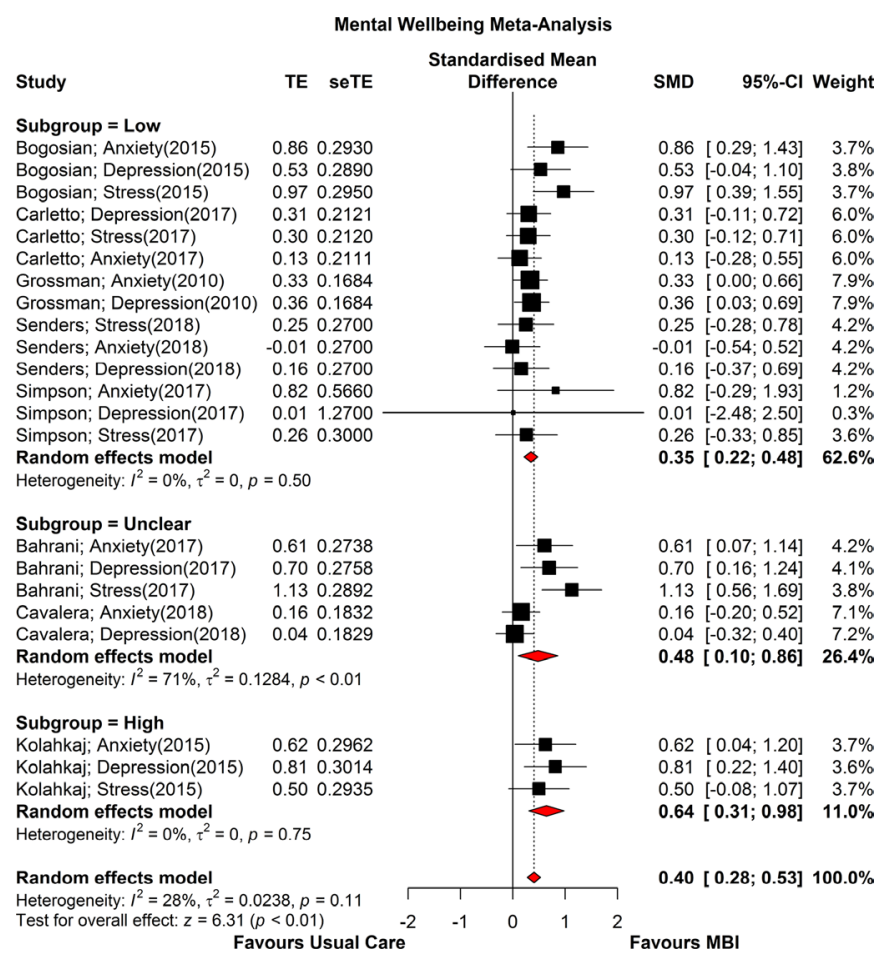

Figure 5 Risk of bias forest plot. MBI, mindfulness-based intervention; $\mathrm{SMD}$, standardised mean difference.
Exercise';22 spasticity during guided progressive muscle relaxation; ${ }^{21}$ anxiety following the MBSR retreat. ${ }^{21}$

\section{DISCUSSION}

Summary of main findings

This systematic review and meta-analysis identified twelve RCTs that assessed MBI effect on mental well-being in PwMS. Only three studies compared an MBI against active comparators, six against usual care and in three this was unclear. Two studies explicitly measured intervention fidelity. Most studies had small sample sizes, but six were powered to detect meaningful effects, follow-up ranging from immediately post-MBI-1 year later.

In total, 744 PwMS took part in these studies, the slight majority $(60 \%)$ having a relapsing phenotype. Where reported, the majority ethnic group was Caucasian, and most participants female. Reporting on levels of comorbidity and disability was mostly poor.

Five studies used MBSR explicitly, two based on MBSR; three MBCT, one MiCBT, one Mindfulness of Movement. The majority of studies were delivered in face-to-face groups. Most studies reported delivering core MBI components and home practices. Class sizes varied. Mostly, teacher characteristics were poorly described. Treatment adherence was reported in seven studies, variably as session attendance \pm home practice. Attrition ranged widely (0\%-39\%). Adverse events appear infrequent but were rarely reported.

Generally, study quality has improved since our last review; ${ }^{8}$ in this current study, five of the RCTs score a low risk of bias on all items in the Cochrane Collaboration tool.

\begin{tabular}{|c|c|c|c|}
\hline Predictors & Estimates & $95 \% \mathrm{Cl}$ & $P$ value \\
\hline Intervention type (MBI) & -0.03 & $(-0.33$ to 0.26$)$ & 0.82 \\
\hline Intervention type (MBCT) & 0.51 & (0.13 to 0.88$)$ & 0.008 \\
\hline Risk of bias (high) & 0.54 & (0.12 to 0.96$)$ & 0.01 \\
\hline Risk of bias (low) & 0.18 & $(-0.13$ to 0.49$)$ & 0.25 \\
\hline
\end{tabular}

Reference for intervention type: MBSR; reference for risk of bias: Unclear. $M B C T$, mindfulness-based cognitive therapy; $\mathrm{MBI}$, mindfulness-based intervention; MBSR, mindfulness-based stress reduction. 
Meta-analysis demonstrated that MBIs are moderately effective for improving mental well-being in PwMS. At present, there is insufficient evidence to recommend any particular MBI over another for PwMS.

\section{Comparison with existing literature}

In this study we found MBIs moderately effective for treating anxiety (SMD 0.35; 0.15-0.55), depression (SMD 0.35; 0.170.53 ) and stress (SMD 0.55; 0.25-0.85) in PwMS. A 2004 meta-analysis $^{33}$ on the use of MBIs in diverse chronic medical conditions reported overall effect sizes (Cohen's d) for mental health of $d=0.50(0.43-0.56)$. A 2010 meta-analysis ${ }^{34}$ on MBSR effects on mental health in patients with varied chronic medical conditions reported smaller effect sizes (Hedge's $g$ ): $g=0.27$ $(0.19-0.35)$ for depression; $g=0.24(0.10-0.38)$ for anxiety; and $g=0.32(0.13-0.50)$ for psychological distress. A 2016 meta-analysis ${ }^{4}$ of interventions for anxiety and depression in PwMS reported small effect sizes for psychological treatments (mostly CBT, $\mathrm{n}=9$, none testing a MBI) (SMD 0.45; 0.16-0.74), medium effects for pharmacological treatments (SMD 0.63; 0.20-1.07) in improving depression, but limited evidence for effective treatments for anxiety.

When compared with our own analysis, accumulating evidence suggests that MBIs are at least moderately effective for treating anxiety, depression and stress in PwMS; effect sizes comparable with CBT, but marginally less effective than medication, for treating depression.

\section{Strengths of this review}

We adopted rigorous search, appraisal and analysis strategies, using a multi-disciplinary team of experienced reviewers for data extraction and a statistician for our meta-analysis. Our methods were guided by the PRISMA checklist, ${ }^{13}$ the TIDieR checklist ${ }^{11}$ and the Cochrane Collaboration tool for assessing risk of bias. ${ }^{12}$

\section{Limitations of this review}

This study only included RCTs, necessarily excluding other important sources of data, such as observational and qualitative studies, particularly useful when considering intervention feasibility, acceptability and accessibility. However, by using validated methods such as SPIO, the TIDieR checklist and Cochrane Collaboration tool for risk of bias, various 'qualitative' aspects of feasibility, replicability and trial conduct were covered.

\section{Strengths and limitations of the included studies}

All studies included in this review were RCTs. However, six had small sample sizes $(n \leq 50)$, only six were powered to detect statistical significance on outcome measures, and only three tested an MBI against an active comparator. One study did not report on participant age $;^{26}$ the extractable mean (SD) age from the remaining studies was relatively low (41.4). This potentially indicates a pooled sample skewed towards lower levels of disability. ${ }^{35}$ Although seven studies stipulated EDSS as an inclusion criterion, only five reported mean (SD) values, making it difficult to determine what role a given MBI may have relative to disability level. While all MS phenotypes featured among the included studies, only two evaluated MBI effects on specific phenotypes, ${ }^{23} 31$ limiting analysis to pooled data, meaning no recommendations can be made for people with a particular type of MS. Participant SES was poorly covered; important because there is an established link between lower SES and higher incidence of depression in those with MS. ${ }^{36}$ Both MBSR and MBCT appear effective, with no clear optimal MBI. Several studies altered the manualised MBSR or MBCT courses, often with little/ no justification, although most included core MBI components.

\section{Implications for research}

Generally, the quality and weight of evidence supporting MBIs to improve mental well-being in PwMS has improved since our previous systematic review. However, many of the RCTs in this meta-analysis did not clearly follow the CONSORT ${ }^{12}$ criteria and scored unclear or high on the Cochrane Collaboration risk of bias ${ }^{10}$ tool. Furthermore, several lacked in clarity when it came to describe the MBI used. By using validated, evidencebased tools such as the CONSORT ${ }^{12}$ and TIDieR ${ }^{11}$ checklists, study authors could improve reporting in this area and help identify key gaps in knowledge and future research priorities.

The optimal MBI for PwMS remains unclear. As per the MRC guidance on complex interventions, ${ }^{6}$ PwMS should help design an optimised MBI and this should then be tested in a definitive RCT against current 'gold-standard' treatment(s). In PwMS who have stress or depression, this would mean testing against a matched group CBT course and usual care.

An additional consideration for future research in this area could be how MBI training may impact on disease activity in PwMS. Systematic review and meta-analytic data suggest a link between perceived stress and MS relapse. ${ }^{37} 38$ Preliminary RCT evidence supports CBT-based stress management therapy having a potential role in diminishing underlying disease activity in MS (gadolinium uptake on MRI). Besides the beneficial effects on perceived stress deriving from CBT, the clinical utility of these findings remains unclear. ${ }^{39}$ However, on the basis of the beneficial effects on perceived stress identified in this meta-analysis, an RCT study examining the effects of MBI training on disease activity in PwMS may now be indicated.

\section{Implications for clinical practice}

MBIs effectively improve mental well-being in PwMS. It remains unclear where an MBI might 'fit' in the bigger picture of managing comorbid mental health conditions in PwMS, where patient characteristics and clinical severity may vary widely, and stepped care models increasingly predominate. ${ }^{40}$ However, on the basis of our study and others, it seems prudent to recommend systematic, group-based MBI training with regular home practice $^{41}$ and follow-up. ${ }^{42}$

\section{CONCLUSIONS}

A substantial body of RCT evidence now exists supporting the use of MBIs in PwMS to improve mental well-being. Study quality is improving, but significant scope for improvement still exists in study design and reporting. What constitutes the optimal MBI for PwMS remains unclear.

Contributors RS, SWM, NR, ML conceived the design of this project. ML carried out the database searches. RS, SS, JB carried out the literature screening and data extraction. NR and RS carried out the meta-analysis. RS led the writing of the manuscript. All authors read and approved the final version of the manuscript.

Funding This study was funded by the R S McDonald Trust (SC012710).

Competing interests None declared.

Patient consent for publication Not required.

Provenance and peer review Not commissioned; externally peer reviewed.

\section{REFERENCES}

1 Malcomson KS, Lowe-Strong AS, Dunwoody L. What can we learn from the personal insights of individuals living and coping with multiple sclerosis? Disabil Rehabil 2008;30:662-74 
2 Simpson RJ, McLean G, Guthrie B, et al. Physical and mental health comorbidity is common in people with multiple sclerosis: nationally representative cross-sectional population database analysis. BMC Neurol 2014;14.

3 Paparrigopoulos T, Ferentinos P, Kouzoupis A, et al. The neuropsychiatry of multiple sclerosis: focus on disorders of mood, affect and behaviour. Int Rev Psychiatry 2010;22:14-21.

4 Fiest KM, Walker JR, Bernstein CN, et al. Systematic review and meta-analysis of interventions for depression and anxiety in persons with multiple sclerosis. Mult Scler Relat Disord 2016;5:12-26.

5 Reynard AK, Sullivan AB, Rae-Grant A. A systematic review of stress-management interventions for multiple sclerosis patients. Int J MS Care 2014;16:140-4.

6 Craig PD, P; Maclntyre S, et. al. Developing and evaluating complex interventions: new guidance. Medical Research Council. Online: Medical Research Council UK, 2008.

7 Goyal M, Singh S, Sibinga EMS, et al. Meditation programs for psychological stress and well-being: a systematic review and meta-analysis. JAMA Intern Med 2014; 174:357-68

8 Simpson R, Booth J, Lawrence $M$, et al. Mindfulness based interventions in multiple sclerosis - a systematic review. BMC Neurol 2014;14.

9 Richardson WS, Wilson MC, Nishikawa J, et al. The well-built clinical question: a key to evidence-based decisions. ACP J Club 1995;123:A12-13.

10 Schulz KF, Altman DG, Moher D. Consort 2010 statement: updated guidelines for reporting parallel group randomised trials. BMC Med 2010;8.

11 Hoffmann TC, Glasziou PP, Boutron I, et al. Better reporting of interventions: template for intervention description and replication (TIDieR) checklist and guide. BMJ 2014;348:g1687.

12 Higgins JPT, Altman DG, Gøtzsche PC, et al. The Cochrane collaboration's tool for assessing risk of bias in randomised trials. BMJ 2011;343:d5928.

13 Moher Det al. Preferred reporting items for systematic reviews and meta-analyses: the PRISMA statement. Ann Intern Med 2009;151:264-9.

14 DerSimonian R, Laird N. Meta-analysis in clinical trials revisited. Contemp Clin Trials 2015:45:139-45.

15 Higgins JPT, Thompson SG, Deeks JJ, et al. Measuring inconsistency in meta-analyses. BMJ 2003:327:557-60.

16 Sterne JA, Egger M, Moher D. Addressing reporting biases. Cochrane handbook for systematic reviews of interventions: Cochrane book series, 2008: 297-333.

17 Egger M, Davey Smith G, Schneider M, et al. Bias in meta-analysis detected by a simple, graphical test. BMJ 1997;315:629-34.

18 Duval S, Tweedie R. TRIM and fill: a simple funnel-plot-based method of testing and adjusting for publication bias in meta-analysis. Biometrics 2000:56:455-63.

19 Duval S. A nonparametric "trim and fill" method of accounting for publication bias in meta-analysis. Journal of the American Statistical Association 2000;95:89-98.

20 Schwarzer G. Meta: an R package for meta-analysis. $R$ news 2007;7:40-5.

21 Senders $A$, Hanes $D$, Bourdette $D$, et al. Impact of mindfulness-based stress reduction for people with multiple sclerosis at 8 weeks and 12 months: A randomized clinical trial. Multiple Sclerosis Journal 2018;328.

22 Simpson R, Mair FS, Mercer SW. Mindfulness-based stress reduction for people with multiple sclerosis - a feasibility randomised controlled trial. BMC Neurol 2017;17.

23 Bahrani S, Zargar F, Yousefipour $G$, et al. The effectiveness of mindfulness-integrated cognitive behavior therapy on depression, anxiety, and stress in females with multiple sclerosis: a single blind randomized controlled trial. Iran Red Crescent Med J 2017;19.
24 Carletto S, Tesio V, Borghi M, et al. The effectiveness of a body-affective mindfulness intervention for multiple sclerosis patients with depressive symptoms: a randomized controlled clinical trial. Front Psychol 2017;8.

25 Amiri M, Rabiei M, Donyavi V. Effectiveness of mindfulness training in enhancing executive function and decreasing symptoms of depression and anxiety in patients with multiple sclerosis (MS). J Behav Brain Sci 2016:06:329-36.

26 Mahdavi A, Yazdanbakhsh K, Sharifi M. The effectiveness of Mindfulness-Based cognitive therapy in reducing psychological symptoms, Meta-Worry and thought fusion of multiple sclerosis patients 2016.

27 Nejati S, Rajezi Esfahani S, Rahmani S, et al. The effect of group mindfulness-based stress reduction and consciousness yoga program on quality of life and fatigue severity in patients with MS. J Caring Sci 2016;5:325-35.

28 Bogosian A, Chadwick P, Windgassen S, et al. Distress improves after mindfulness training for progressive MS: a pilot randomised trial. Multiple Sclerosis Journal 2015;21:1184-94.

29 Kolahkaj B, Zargar F. Effect of Mindfulness-Based stress reduction on anxiety, depression and stress in women with multiple sclerosis. Nurs Midwifery Stud 2015;4.

30 Grossman P, Kappos L, Gensicke H, et al. MS quality of life, depression, and fatigue improve after mindfulness training: a randomized trial. Neurology 2010;75:1141-9.

31 Mills N, Allen J. Mindfulness of movement as a coping strategy in multiple sclerosis. A pilot study. Gen Hosp Psychiatry 2000;22:425-31.

32 Cavalera C, Rovaris M, Mendozzi L, et al. Online meditation training for people with multiple sclerosis: a randomized controlled trial. Multiple Sclerosis Journal 2018;1352458518761187.

33 Grossman P, Niemann L, Schmidt S, et al. Mindfulness-based stress reduction and health benefits. A meta-analysis. J Psychosom Res 2004;57:35-43.

34 Bohlmeijer $E$, Prenger $R$, Taal $E$, et al. The effects of mindfulness-based stress reduction therapy on mental health of adults with a chronic medical disease: a metaanalysis. J Psychosom Res 2010;68:539-44.

35 Compston A, Coles A, Sclerosis M. Multiple sclerosis. Lancet 2008:372:1502-17.

36 Marrie RA, Horwitz R, Cutter G, et al. The burden of mental comorbidity in multiple sclerosis: frequent, underdiagnosed, and undertreated. Mult Scler 2009;15:385-92.

37 Mohr DC, Hart SL, Julian L, et al. Association between stressful life events and exacerbation in multiple sclerosis: a meta-analysis. BMJ 2004;328.

38 Artemiadis AK, Anagnostouli MC, Alexopoulos EC. Stress as a risk factor for multiple sclerosis onset or relapse: a systematic review. Neuroepidemiology 2011;36:109-20.

39 Mohr DC, Lovera J, Brown T, et al. A randomized trial of stress management for the prevention of new brain lesions in MS. Neurology 2012;79:412-9.

40 Clark DM. Implementing NICE guidelines for the psychological treatment of depression and anxiety disorders: the IAPT experience. Int Rev Psychiatry 2011;23:318-27

41 Parsons CE, Crane C, Parsons LJ, et al. Home practice in Mindfulness-Based cognitive therapy and Mindfulness-Based stress reduction: a systematic review and metaanalysis of participants' mindfulness practice and its association with outcomes. Behav Res Ther 2017;95:29-41.

42 Mathew KL, Whitford HS, Kenny MA, et al. The long-term effects of mindfulness-based cognitive therapy as a relapse prevention treatment for major depressive disorder. Behav Cogn Psychother 2010;38:561-76. 